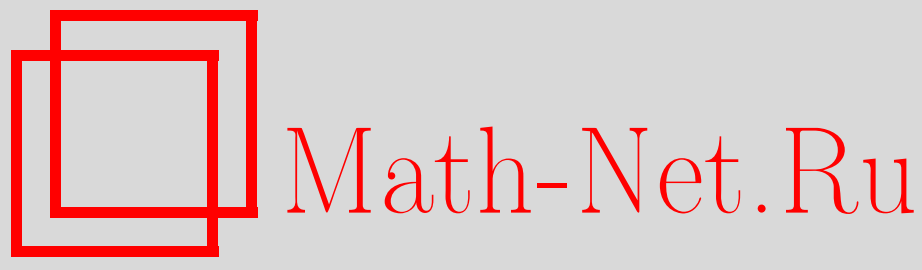

Общероссийский математический портал

В. Г. Звягин, О множестве критических значений потенциальных фредгольмовых функционалов, Матем. заметки, 1998, том 63, выпуск 1, 133-135

DOI: https://doi.org/10.4213/mzm1256

Использование Общероссийского математического портала Math-Net.Ru подразумевает, что вы прочитали и согласны с пользовательским соглашением http://www.mathnet.ru/rus/agreement

Параметры загрузки: 
IP : 3.85 .73 .92

26 апреля 2023 г., 10:03:24

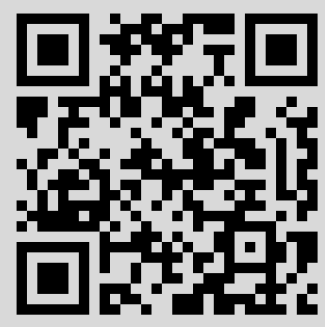




\section{О МНОЖЕСТВЕ КРИТИЧЕСКИХ ЗНАЧЕНИЙ ПОТЕНЦИАЛЬНЫХ ФРЕДГОЛЬМОВЫХ ФУНКЦИОНАЛОВ}

\section{В.Г. Звягин}

1. Хорошо известна роль, которую играет для исследования различных классов отображений тот факт, что множество критических значений для отображений из этого класса имеет меру нуль (см., например, [1], [2]). В конечномерном анализе этот факт носит название теоремы Морса-Сарда. В бесконечномерном случае имеется обобщение этого резултата на класс фредгольмовых отображений [3]. Однако, для класса гладких функционалов $V: E \rightarrow \mathbb{R}$, определенных на вещественных бесконечномерных банаховых пространствах $C^{\infty}$, этот факт в общем случае неверен. Соответствуюшие примеры имеются в [2], [4]. Тем не менее, при дополнительном условии фредгольмовости отображения $D^{2} V(x): E \rightarrow E^{*}$ для каждого $x \in E$, сепарабельности и рефлексивности пространства $E$ аналог теоремы Морса-Сарда имеет место. Это показано в работе [5].

Однако, в приложениях (см., например, [6]) часто возникают $C^{\infty}$-гладкие функционалы, определенные, во-первых, на нерефлексивных банаховых пространствах, и, во-вторых, вторая производная которых не обладает свойством фредгольмовости, как отображение из $E$ в $E^{*}$, хотя это свойство имеет место для некоторых аналогов второй производной, но уже в паре банаховых пространств. Здесь рассматривается такой класс функционалов и для него доказывается аналог теоремы Морса-Сарда.

2. Итак, пусть $E$ - вещественное банахово пространство, $V: E \rightarrow \mathbb{R}-C^{2}$-гладкий функционал на $E$.

Будем предполагать, что выполнены следующие условия:

1) задана такая пара вещественных линейных пространств $F, H$, где $F$ банахово, а $H$ гильбертово, что $E$ линейно и непрерывно вложено в $F, F$ линейно и непрерьвно вложено в $H$, причем суперпозиция этих вложений $i: E \rightarrow H$ является плотньм вложением $E$ в $H$;

2) задано $C^{1}$-гладкое отображение $f: E \rightarrow F$ такое, что

$$
V^{\prime}(x) h=\langle f(x), h\rangle \quad \forall x \in E, \quad \forall h \in i(E),
$$

где $\langle\cdot, \cdot\rangle$ - скалярное произведение в $H$. 
Отображение $f$ назьвается градиентом функционала $V$ и обозначается символом $\operatorname{grad}_{H} V$. Соответственно $V$ называют потенциалом отображения $f$ или потенциальным функционалом.

Напомним (см., например, [7]), что $C^{1}$-гладкое отображение $f: E \rightarrow F$ называется фредгольмовым отображением индекса нуль, если производная $f^{\prime}(x): E \rightarrow F$ в каждой точке $x \in E$ является линейньм фредгольмовым оператором индекса нуль, т.е. $\operatorname{dim} \operatorname{Ker} f^{\prime}(x)=\operatorname{dim} \operatorname{Coker} f^{\prime}(x)<\infty$.

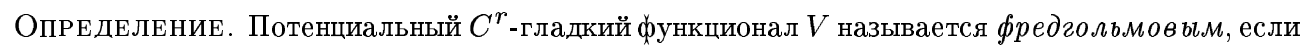
его градиент $f=\operatorname{grad}_{H} V: E \rightarrow F$ является фредгольмовым отображением индекса нуль.

Ряд примеров потенциальных фредгольмовых функционалов, возникающих в различных вопросах механики, можно найти в [6].

Далее, точка $x \in E$ называется критической точкой $C^{1}$-гладкого функционала $V: E \rightarrow \mathbb{R}$, если $V^{\prime}(x)=0$. Обозначим через $B$ множество критических точек функционала $V$. Тогда их образ $V(B)$ назьвается множсеством критических значений этого функционала.

Теперь сформулируем основной резултат.

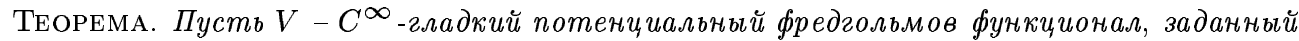
на сепарабельном вещественном банаховом пространстве Е. Тогда мнохество критических значений функчионала $V$ имеет лебегову меру нуль.

ДоКАЗАтЕльСтво. Рассмотрим множество $B$ критических точек функционала $V$, т.е. $B=$ $\left\{x \in E: V^{\prime}(x)=0\right\}$.

Пусть $\widetilde{x} \in B$ и $A=f^{\prime}(\widetilde{x})$. Так как $A$ является линейным фредгольмовым оператором индекса нуль, имеет место разложение (см. [7]) $E=\operatorname{Ker} A \times E_{2}, F=\operatorname{Im} A \times F_{2}$, где $E_{2}, F_{2}$ - подпространства соответственно пространств $E$ и $F$, причем $F_{2}$ изоморфно Coker $A$.

Тогда отображение $f: \operatorname{Ker} A \times E_{2} \rightarrow \operatorname{Im} A \times F_{2}$ можно записать в следующей координатной форме: $f\left(x_{1}, x_{2}\right)=\left(f_{1}\left(x_{1}, x_{2}\right), f_{2}\left(x_{1}, x_{2}\right)\right)$, где $x_{1} \in \operatorname{Ker} A, x_{2} \in E_{2}, f_{1}\left(x_{1}, x_{2}\right) \in \operatorname{Im} A$, $f_{2}\left(x_{1}, x_{2}\right) \in F_{2}$. Определим отображение $\alpha: \operatorname{Ker} A \times E_{2} \rightarrow \operatorname{Ker} A \times \operatorname{Im} A$ по формуле $\alpha\left(x_{1}, x_{2}\right)=$ $\left(x_{1}, f_{1}\left(x_{1}, x_{2}\right)\right), x_{1} \in \operatorname{Ker} A, x_{2} \in E_{2}$. Отображение $\alpha$ является $C^{\infty}$-гладким отображением, причем $\alpha^{\prime}(\widetilde{x})$ является изоморфизмом соответствующих пространств. По теореме об обратном отображении $\alpha$ - локальный $C^{\infty}$-диффеоморфизм в точке $\widetilde{x}$, т.е. существуют окрестности $U$ точки $\widetilde{x}$ и $W$ точки $\alpha(\widetilde{x})$ такие, что $\left.\alpha\right|_{U}: U \rightarrow W$ есть $C^{\infty}$-диффеоморфизм. Без ограничения общности можно считать, что $W=W_{1} \times W_{2}$, где $W_{1} \subseteq \operatorname{Ker} A, W_{2} \subseteq \operatorname{Im} A$.

Определим функционал $\widetilde{V}: W \rightarrow \mathbb{R}$ по формуле $\widetilde{V}\left(w_{1}, w_{2}\right)=V \alpha^{-1}\left(w_{1}, w_{2}\right), w_{1} \in W_{1}, w_{2} \in W_{2}$. Очевидно, функционал $V$ является $C^{\infty}$-гладким как суперпозиция двух $C^{\infty}$-гладких отображений. Далее, поскольку $\left(\alpha^{-1}\right)^{\prime}(\alpha(\widetilde{x}))$ - изоморфизм, уменьшая $W$ в случае необходимости, можно считать, что $\left(\alpha^{-1}\right)^{\prime}\left(w_{1}, w_{2}\right)$ также является изоморфизмом для всех $\left(w_{1}, w_{2}\right) \in W$. Учитывая это, заметим, что из равенства $\widetilde{V}^{\prime}(w)=V^{\prime}\left(\alpha^{-1}(w)\right) \cdot\left(\alpha^{-1}\right)^{\prime}(w)$ следует взаимно-однозначное соответствие между множеством $\widetilde{C}$ критических точек функционала $\widetilde{V}$ в окрестности $W$ и множеством $C$ критических точек функционала $V$ на множестве $U$, причем это соответствие осуществляется отображением $\alpha^{-1}$.

Заметим также, что множество $\widetilde{C}$ принадлежит подпространству $\operatorname{Ker} A$. В самом деле, пусть $w$ - критическая точка функционала $V^{\prime}$. Ей соответствует критическая точка функционала $V$ $x=\alpha^{-1}(w)$, т.е. $V^{\prime}(x)=0$. Но тогда $f(x)=0$. Следовательно, и ее первая компонента $f_{1}(x)=0$. Поэтому $\alpha(x)=\alpha\left(x_{1}, x_{2}\right)=\left(x_{1}, f_{1}(x)\right)=\left(x_{1}, 0\right)=w \in \operatorname{Ker} A$.

Итак, пусть $V(C)$ - множество критических значений функционала $\left.V\right|_{U}$ и $\widetilde{V}(\widetilde{C})$ - множество критических значений функционала $\widetilde{V}$. В силу предыдущих рассуждений, имеем $V(C)=\widetilde{V}(\widetilde{C})$.

Выше показано, что $\widetilde{C} \subseteq \operatorname{Ker} A$ и, следовательно, для $w \in \widetilde{C}$ имеет место представление $w=$ $\left(w_{1}, 0\right)$, где $w_{1} \in W_{1}$ и $\widetilde{V}(\bar{w})=\widetilde{V}\left(w_{1}, 0\right)$.

Определим функцию $\varphi: W_{1} \rightarrow \mathbb{R}$ по формуле $\varphi\left(w_{1}\right)=\widetilde{V}\left(w_{1}, 0\right)$. Обозначим через $C_{0}$ множество критических точек функционала $\varphi$ на множестве $W_{1}$. Очевидно, что $C \subseteq \widetilde{C}_{0}$ и, следовательно, $\widetilde{V}(\widetilde{C}) \subseteq \varphi\left(C_{0}\right)$. 
Функция $\varphi: W_{1} \rightarrow \mathbb{R}$ принадлежит классу $C^{\infty}$ и, следовательно, по теореме Морса-Сарда лебегова мера $\mu\left(\varphi\left(C_{0}\right)\right)=0$. Но тогда $\mu(\widetilde{V}(\widetilde{C}))=0$. Откуда имеем $\mu(V(C))=0$.

Таким образом, каждой точке $x \in B$ соответствует окрестность $U$ такая, что множество $V(B \cap U)$ имеет лебегову меру нуль. Окрестности типа $U$ покрывают множество $B$. Из этого покрытия выделим счетное подпокрытие, что возможно в силу сепарабельности пространства $E$. Поскольку счетное объединение множеств меры нуль имеет меру нуль, то множество $f(B)$ имеет меру нуль. Теорема доказана.

\section{СПИСОК ЦИТИРОВАННОЙ ЛИТЕРАТУРЫ}

1. Abraham R., Robbin J. Transversal Mappings and Flows. New York: Benjamin, 1967. 2. Иллс Дж. // УМН. 1969. Т. 24. № 3. С. 157-210. 3. Smale S. // Amer. J. Math. 1965. V. 87. № 4. P. 861-866. 4. Kupka J. // Proc. Amer. Math. Soc. 1965. V. 16. № 5. P. 954-957. 5. Похожаев С. И. // Матем. сб. 1968. Т. 75 (117). №1. С. 106-111. 6. Сапронов Ю. И. // УМН. 1996. Т. 51. №1. С. 101-132. 7. Борисович Ю. Г., Звягин В. Г., Сапронов Ю. И. // УМН. 1977. T. 32. № 4. C. $3-54$.

Воронежский государственный университет

Поступило

E-mail: zvyagin@alg.vsu.ru 